Acta Crystallographica Section A

Foundations of Crystallography

ISSN 0108-7673

Received 22 January 2007

Accepted 9 March 2007

(C) 2007 International Union of Crystallography Printed in Singapore - all rights reserved

\section{A simple method of determining the form of the phonon part of tensors describing quasicrystal properties}

\author{
Hans Grimmer \\ Laboratory for Development and Methods, Condensed Matter Research with Neutrons and Muons, \\ Paul Scherrer Institut, CH-5232 Villigen PSI, Switzerland. Correspondence e-mail: \\ hans.grimmer@psi.ch
}

\begin{abstract}
It is shown that the restrictions on the form of property tensors of rank $<5$ that follow from the Neumann principle for the point groups describing quasicrystals can easily be deduced from the restrictions for the point groups describing ordinary crystals. For octagonal and dodecagonal point groups, this is true even for property tensors of rank $<8$ and $<12$, respectively. The results derived in a number of papers for various physical properties of quasicrystals with certain point-group symmetries are generalized to all quasicrystal point groups, and it is shown that the results become more lucid if the classification of quasicrystal point groups with a principal axis into pentagonal, decagonal, octagonal and dodecagonal ones is done appropriately. A comparison with other approaches shows that applying the Neumann principle for the point groups describing quasicrystals yields the form of the so-called phonon part of the tensor under consideration. Connections between the restrictions valid for property tensors of arbitrary rank are given for general Heesch-Shubnikov point groups in three dimensions.

\section{Introduction}

The rotational symmetry of a right prism or antiprism with a regular $n$-gon as basis is $n 2$ if $n$ is odd, $n 22$ if $n$ is even; taking the inversion $\overline{1}$ as an additional generator, the centrosymmetric groups $\bar{n} m$ ( $n$ odd) and $n / \mathrm{mmm}(n$ even) are obtained. The pentagonal and decagonal point groups are (proper or improper) subgroups of $10 / \mathrm{mmm}$ containing fivefold rotations; those groups that are subgroups of $\overline{5} m$ are called pentagonal, the others decagonal. Similarly, the octagonal point groups are those subgroups of $8 / \mathrm{mmm}$ containing fourfold rotations that are not subgroups of $4 / \mathrm{mmm}$; the dodecagonal point groups are those subgroups of $12 / \mathrm{mmm}$ containing sixfold rotations that are not subgroups of $6 / \mathrm{mmm}$. The five pentagonal, seven octagonal, seven decagonal and seven dodecagonal point groups are listed in Table 1 below [see also Table 3-1 of Steurer (2004) or Table 10.1.4.2 of Hahn \& Klapper (2002)].

Since the discovery of quasicrystals, several papers have been published giving the restrictions on the form of property tensors that follow from the Neumann principle for quasicrystal point groups [see e.g. Jiang et al. (1995) or Rama Mohana Rao \& Hemagiri Rao $(1992,1993)]$. Influenced by the Schoenflies notation, these authors define pentagonal, decagonal, octagonal and dodecagonal not as described above (see Table 2 in Jiang et al., 1995). It will be shown that their definition obscures certain features of the dependence of the form of property tensors on the point group of the quasicrystal.

A theorem due to Hermann (1934) states that for all tensors of rank $n$ a symmetry rotation of order $>n$ leads to the same restrictions as a symmetry rotation of infinite order. Notice that a rotation-inversion axis $\bar{n}$ contains a rotation axis $n$ if $n$ is odd, but only $n / 2$ if $n$ is even. It follows that for tensors of rank $<5$ the pentagonal, decagonal, dodecagonal and octagonal point groups (except $\overline{8}$ and $\overline{8} 2 \mathrm{~m}$ ) lead to the same restrictions as the corresponding hexagonal groups (except $\overline{6}$ and $\overline{6} \mathrm{~m} 2$ ) and that properties of icosahedral crystals described by tensors of rank $<5$ are isotropic.

\section{Point-group symmetries of quasicrystals; general results on the form of property tensors}

Let me start by considering the classical (i.e. monochrome) point groups. Table 1 lists the trigonal, pentagonal, hexagonal, octagonal, decagonal, dodecagonal and icosahedral point groups and their classification into Laue classes: all groups $G$ for which the smallest centrosymmetric group containing $G$ is the same belong to one Laue class. In Table 1, the centrosymmetric group of each Laue class appears in the bottom line, whereas the pure rotation group appears in the top line. The Laue classes of the pentagonal, octagonal, decagonal and 
Table 1

Distribution of trigonal, pentagonal, hexagonal, octagonal, decagonal, dodecagonal and icosahedral point groups into Laue classes.

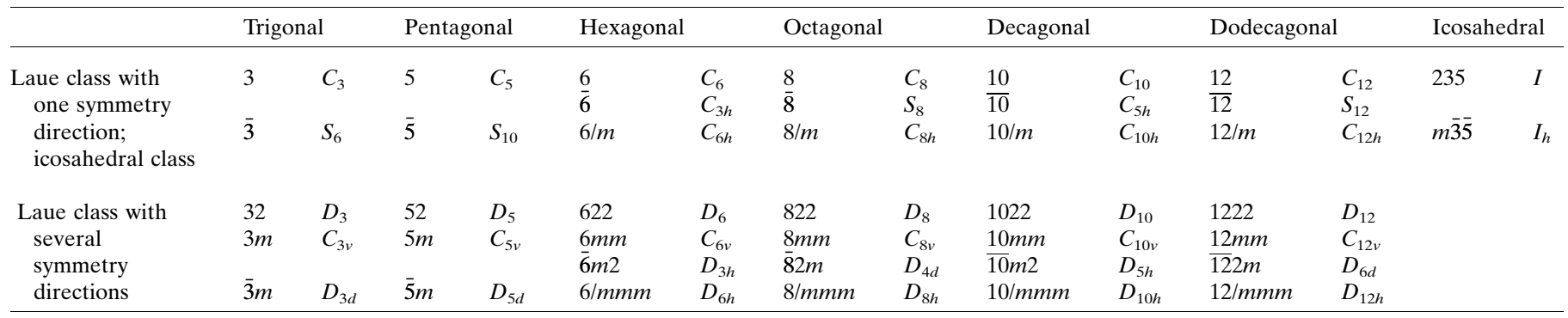

dodecagonal systems will be referred to as $\mathrm{L} n_{1}$ and $\mathrm{L} n_{2}$, where $n=5,8,10$ and 12 , respectively, and where the subscript 1 refers to the Laue class containing one symmetry direction, the subscript 2 to the class with several symmetry directions.

Polar tensors of even rank and axial tensors of odd rank are invariant under space inversion $\overline{1}$; we say that they have positive parity. For such property tensors, the restrictions following from the Neumann principle depend only on the Laue class of the point group. Therefore, it suffices to determine the restrictions for the pure rotation group in the Laue class under consideration.

Polar tensors of odd rank and axial tensors of even rank change sign under space inversion $\overline{1}$; we say that they have negative parity. Such property tensors vanish for the centrosymmetric groups, whereas the restrictions following from the Neumann principle for the pure rotation groups do not depend on the behaviour of the tensor under $\overline{1}$. It has been shown by Grimmer (1991) that the restrictions for the remaining crystallographic point groups can easily be deduced from those for the pure rotation groups. Let me show how this result can be extended to the icosahedral, pentagonal, decagonal, dodecagonal and octagonal groups. The right-handed Cartesian coordinate system used for describing the form of tensors has its axes along twofold symmetry axes in the case of icosahedral symmetry; for the $n$-gonal symmetries, it has $z$ along the principal symmetry direction and $x$ along a binary symmetry direction if such symmetry directions exist. For $n$-gonal symmetries, we define a right-handed quasicrystal coordinate system $\mathbf{a}, \mathbf{b}, \mathbf{c}$ with $\mathbf{c}$ along the principal symmetry direction, $\mathbf{a}$ and $\mathbf{b}$ normal to $\mathbf{c}$, and along binary symmetry directions if such symmetry directions exist. The first entry in the Hermann-Mauguin symbol corresponds to $\mathbf{c}$, the second to $\mathbf{a}$, the angle between $\mathbf{a}$ and $\mathbf{b}$ will be chosen below for the various cases such that the third entry in the Hermann-Mauguin symbol corresponds to $\mathbf{a}-\mathbf{b}$.

For the pentagonal and decagonal groups, we choose $\mathbf{a}$ and $\mathbf{b}$ at an angle of $144^{\circ}$. It follows that $\mathbf{a}-\mathbf{b}$ makes an angle of $18^{\circ}$ with $\mathbf{a}$. The restrictions on the form of a property tensor with negative parity imposed by $5 \mathrm{~m}$ are such that each tensor satisfying the restrictions for 5 can be decomposed uniquely into a tensor satisfying the restrictions for 52 and a tensor satisfying the restrictions for $5 \mathrm{~m}$. Symbolically, we may write: $T[5]=T[52] \oplus T[5 m]$. Analogously: $T[10]=$
$T[1022] \oplus T[10 \mathrm{~mm}], \quad T[5]=T[10] \oplus T[\overline{10}], \quad T[52]=$ $T[1022] \oplus T[\overline{10} 2 m]$. Finally, $T[\overline{10} \mathrm{~m} 2]$ may be obtained in two different ways, providing a check of the calculation: $T[5 \mathrm{~m}]=$ $T[10 \mathrm{~mm}] \oplus T[\overline{10} \mathrm{~m} 2]$ and $T[\overline{10}]=T[\overline{10} 2 \mathrm{~m}] \oplus T[\overline{10} \mathrm{~m} 2]$. All these equations follow from the facts that the two oriented point groups on the right-hand side $(a)$ have the point group on the left-hand side in common and $(b)$ together generate a centrosymmetric point group (for which $T$ vanishes).

Consider as an example the tensor that describes the piezoelectric effect. It has rank 3 and is symmetric in its last two indices. Following Nye (1985), it can be characterized by a $3 \times 6$ matrix $d_{i j}$. We shall see below that for 5 it has the same form as for 6 , for 52 the same as for 622 . According to Nye (1985), we then have

$$
\begin{aligned}
d_{i j}[5] & =\left(\begin{array}{cccccc}
0 & 0 & 0 & d_{14} & d_{15} & 0 \\
0 & 0 & 0 & d_{15} & -d_{14} & 0 \\
d_{31} & d_{31} & d_{33} & 0 & 0 & 0
\end{array}\right), \\
d_{i j}[52] & =\left(\begin{array}{cccccc}
0 & 0 & 0 & d_{14} & 0 & 0 \\
0 & 0 & 0 & 0 & -d_{14} & 0 \\
0 & 0 & 0 & 0 & 0 & 0
\end{array}\right) .
\end{aligned}
$$

It follows from $T[5]=T[52] \oplus T[5 m]$ that

$$
d_{i j}[5 m]=\left(\begin{array}{cccccc}
0 & 0 & 0 & 0 & d_{15} & 0 \\
0 & 0 & 0 & d_{15} & 0 & 0 \\
d_{31} & d_{31} & d_{33} & 0 & 0 & 0
\end{array}\right) .
$$

For the dodecagonal groups, we choose $\mathbf{a}$ and $\mathbf{b}$ at an angle of $150^{\circ}$. It follows that $\mathbf{a}-\mathbf{b}$ makes an angle of $15^{\circ}$ with $\mathbf{a}$. $T[12]=T[1222] \oplus T[12 \mathrm{~mm}], T[6]=T[12] \oplus T[\overline{12}], T[622]=$ $T[1222] \oplus T[\overline{12} 2 m]$. Finally, $T[\overline{12} \mathrm{~m} 2]$ may be obtained in two different ways, providing a check of the calculation: $T[6 \mathrm{~mm}]=$ $T[12 \mathrm{~mm}] \oplus T[\overline{12} \mathrm{~m} 2]$ and $T[\overline{12}]=T[\overline{12} 2 \mathrm{~m}] \oplus T[\overline{12} \mathrm{~m} 2]$.

For the octagonal groups, we choose $\mathbf{a}$ and $\mathbf{b}$ at an angle of $135^{\circ}$. It follows that $\mathbf{a}-\mathbf{b}$ makes an angle of $22.5^{\circ}$ with $\mathbf{a}$. $T[8]=T[822] \oplus T[8 \mathrm{~mm}], \quad T[4]=T[8] \oplus T[\overline{8}], \quad T[422]=$ $T[822] \oplus T[\overline{8} 2 m]$. Also, $T[\overline{8} m 2]$ may be obtained in two different ways: $T[4 \mathrm{~mm}]=T[8 \mathrm{~mm}] \oplus T[\overline{8} \mathrm{~m} 2]$ and $T[\overline{8}]=$ $T[\overline{8} 2 m] \oplus T[\overline{8} m 2]$.

Notice that $\overline{8} 2 m$ and $\overline{8} m 2$ differ only in their orientation: they are rotated by $22.5^{\circ}$ with respect to each other; the Schoenflies symbol is $D_{4 d}$ for both. According to $T[\overline{8}]=$ $T[\overline{8} 2 m] \oplus T[\overline{8} m 2]$, the two forms $T[\overline{8} 2 m]$ and $T[\overline{8} m 2]$ will 
differ for tensors of negative parity unless they vanish. Because $T[\overline{8} 2 \mathrm{~m}]$ and $T[\overline{8} \mathrm{~m} 2]$ have the same number of independent components, the number of independent components of $T[\overline{8}]$ will be even. Similar results hold for $\overline{10} 2 \mathrm{~m}$ and $\overline{10} \mathrm{~m} 2$, which are rotated by $18^{\circ}$ with respect to each other, the Schoenflies symbol being $D_{5 h}$ for both, and for $\overline{12} 2 \mathrm{~m}$ and $\overline{12} \mathrm{~m} 2$, which are rotated by $15^{\circ}$ with respect to each other, the Schoenflies symbol being $D_{6 d}$ for both. The possibility of distinguishing the two orientations in which these groups occur in the centrosymmetric group of their Laue class is an essential advantage of the Hermann-Mauguin notation.

The relations given above hold for tensors of arbitrary rank. For tensors of sufficiently low rank, the situation is even simpler thanks to the following theorem by Hermann (1934): a symmetry rotation of order $n$ leads to the same restrictions as a rotation of infinite order for all tensors of rank $<n$. Because all pentagonal and decagonal point groups contain a rotation axis of order 5 , it follows for property tensors of rank $<5$ that for the point groups 5 and 10 we have the same restrictions as for 6 , for 52 and 1022 the same as for 622 , for $5 \mathrm{~m}$ and $10 \mathrm{~mm}$ the same as for $6 \mathrm{~mm}$. Because $T[5]$ and $T[10]$ are the same, it follows in the case of negative parity from $T[5]=$ $T[10] \oplus T[\overline{10}]$ that $T[\overline{10}]$ vanishes, whence also $T[\overline{10} 2 \mathrm{~m}]$ and $T[\overline{10} m 2]$ vanish because they contain $T[\overline{10}]$ as a subgroup.

Similarly, all dodecagonal point groups contain a rotation axis of order 6 (in most cases even of order 12). It follows for tensors of rank $<6$ that for 12 we have the same restrictions as for 6 , for 1222 the same as for 622 , for $12 \mathrm{~mm}$ the same as for $6 \mathrm{~mm}$. Because $T[6]$ and $T[12]$ are the same, it follows in the case of negative parity that $T[\overline{12}]$ vanishes, whence also $T[\overline{12} 2 m]$ and $T[\overline{12} m 2]$ vanish. (Notice that $T[\overline{6}], T[\overline{6} 2 m]$ and $T[\overline{6} \mathrm{~m} 2]$ need not vanish for tensors of negative parity with rank $\geq 3$ because $\overline{6}$ contains a rotation axis of order 3 but not of order 6.)

All octagonal point groups contain a rotation axis of order 4 (in most cases even of order 8). It follows for tensors of rank $<6$ that for 8 we have the same restrictions as for 6 , for 822 the same as for 622 , for $8 \mathrm{~mm}$ the same as for $6 \mathrm{~mm}$. For tensors of rank $<4$, it follows that for 8 we have the same restrictions as for 4 , for 822 the same as for 422 , for $8 \mathrm{~mm}$ the same as for $4 \mathrm{~mm}$. Because for tensors of rank $<4 T[4]$ and $T[8]$ are the same, it follows in the case of negative parity that $T[\overline{8}]$ vanishes, whence also $T[\overline{8} 2 m]$ and $T[\overline{8} m 2]$ vanish.

Finally, it follows from the Neumann principle that properties described by tensors of rank $<5$ must be isotropic in crystals with icosahedral symmetry.

\section{Comparison with results given in the literature on properties of quasicrystals}

Jiang et al. (1990) considered the two icosahedral, five pentagonal and two $(\overline{10}$ and $\overline{10} \mathrm{~m} 2)$ of the seven decagonal point groups. In their Table 1, they consider the piezoelectric tensor (of negative parity). Without making use of the results of Nye (1985), they find for 5 the same form as Nye gives for 6, for 52 the same as Nye for 622 , for $5 m$ the same as Nye for $6 \mathrm{~mm}$, and that the tensor vanishes in the six other cases. The three non-vanishing forms immediately follow from our general results above. Vanishing follows in the cases $\overline{10}$ and $\overline{10} \mathrm{~m} 2$ from our general results above, in the cases $\overline{5}, \overline{5} \mathrm{~m}$ and $m \overline{3} \overline{5}$ because of centrosymmetry; in the cases 235 and $m \overline{3} \overline{5}$ because of isotropy. [Already the cubic symmetry 432 leads to isotropy for tensors of rank <4. Nye (1985) states that the piezoelectric tensor vanishes for 432 , i.e. in the isotropic case.] In their Table 2, Jiang et al. (1990) consider the photoelastic and elastic tensors (of positive parity). Without making use of the results of Nye (1985), they find for the Laue class containing 5 and for $\overline{10}$ the same form as Nye gives for 6 , for the Laue class containing 52 and for $\overline{10} \mathrm{~m} 2$ the same form as Nye gives for 622, and for the icosahedral Laue class the same form as Nye gives for the isotropic case. Also these results immediately follow from our results above, which further show that the forms valid for $\overline{10}$ and $\overline{10} \mathrm{~m} 2$ are valid for all point groups in the corresponding Laue classes. Notice that the elastic tensor has the same form for 6 and for 622 and therefore for $n, n 2$ ( $n$ odd), $n 22$ ( $n$ even) whenever $n \geq 5$. This form is called 'transversely isotropic' (see e.g. Walpole, 1984).

Jiang et al. (1992) considered six of the seven dodecagonal point groups ( $\overline{12} 2 \mathrm{~m}$ is missing). The forms of the piezoelectric, elastic and photoelastic tensors are given in their Tables 2 and 3. Also, these results immediately follow from the results of Nye (1985) combined with the general results in our $\$ 2$. These results also show that their result for the photoelastic tensor with eight free parameters is valid for $\mathrm{L} 12_{1}$, their result with six free parameters for $\mathrm{L}_{2} 2_{2}$, information that is missing in Jiang et al. (1992).

The ordinary Hall effect in its narrow sense is described by an axial tensor of third rank, antisymmetric in its first two indices, invariant not only under space inversion but also under time reversal (see Grimmer, 1993). An axial tensor of third rank, antisymmetric in its first two indices is equivalent to a polar second rank tensor without internal symmetry. Nye (1985) gives its forms in his Table 14. Its non-vanishing coefficients are $R_{11}=R_{22}, R_{33}, R_{21}=-R_{12}$ for point group $3, R_{11}=$ $R_{22}, R_{33}$ for 32 , and $R_{11}=R_{22}=R_{33}$ for the cubic group 23 . Because for second-rank tensors a rotation of order $>2$ has the same effect as a rotation of infinite order and because we deal with a tensor of positive parity, it follows that the form for 3 is valid also for $\mathrm{L} n_{1}$, the form for 32 also for $\mathrm{Ln}_{2}(n=5,8,10$ or 12). The cubic group 23 having more than one rotation of order $>2$ gives rise to an isotropic tensor, valid also for the icosahedral Laue class. Considering the ordinary Hall effect, Jiang et al. (1995) determined for quasicrystals the form of an axial tensor of third rank, antisymmetric in its first two indices. The result, given in their Table 2, is equivalent to the one we obtained above by combining results of Nye (1985) with our general considerations.

The third-order elastic stiffnesses are described by a polar tensor of rank 6, symmetric in the first pair, the second pair and the third pair of indices and symmetric under all permutations of the three pairs. Therefore, it is usually characterized by coefficients $c_{\lambda \mu \nu}$, where $\lambda, \mu$ and $v$ run from 1 to 6 and satisfy $\lambda \leq \mu \leq v$ (see e.g. Landolt-Börnstein, 1979). The restrictions on $c_{\lambda \mu \nu}$ that follow from the Neumann principle 
Table 2

The various non-vanishing forms of the $3 \times 6$ matrix describing piezoelectricity and piezomagnetism in quasicrystals and the corresponding point groups.

The groups within brackets differ only by their orientation. Column 3 gives the form of the vector describing the pyroelectric effect for the point groups in column 1 and the pyromagnetic effect for the point groups in column 4. Pyro- and piezoelectricity vanish for the pentagonal, octagonal, decagonal and dodecagonal groups that do not appear in column 1, pyro- and piezomagnetism for those that do not appear in column 4; all four effects vanish for the icosahedral groups.

\begin{tabular}{|c|c|c|c|}
\hline 1 & 2 & 3 & 4 \\
\hline $\begin{array}{l}\text { Point group in case of } \\
\text { piezoelectricity and pyroelectricity }\end{array}$ & $\begin{array}{l}\text { Form of the } 3 \times 6 \text { matrix describing } \\
\text { the piezo-effects }\end{array}$ & Pyrovector & $\begin{array}{l}\text { Point group in case of piezomagnetism } \\
\text { and pyromagnetism }\end{array}$ \\
\hline $\begin{array}{l}5,51^{\prime} \\
n, n^{\prime}, n 1^{\prime} \\
\text { where } n=8,10 \text { or } 12\end{array}$ & $\left(\begin{array}{cccccc}0 & 0 & 0 & d_{14} & d_{15} & 0 \\
0 & 0 & 0 & d_{15} & -d_{14} & 0 \\
d_{31} & d_{31} & d_{33} & 0 & 0 & 0\end{array}\right)$ & $\left(\begin{array}{l}0 \\
0 \\
p\end{array}\right)$ & $\begin{array}{l}5, \overline{5} \\
n, \bar{n}, n / m \\
\text { where } n=8,10 \text { or } 12\end{array}$ \\
\hline $\begin{array}{l}52,52^{\prime}, 521^{\prime} \\
n 22, n 2^{\prime} 2^{\prime},\left[n^{\prime} 2^{\prime} 2, n^{\prime} 22^{\prime}\right], n 221^{\prime}, \\
\text { where } n=8,10 \text { or } 12\end{array}$ & $\left(\begin{array}{cccccc}0 & 0 & 0 & d_{14} & 0 & 0 \\
0 & 0 & 0 & 0 & -d_{14} & 0 \\
0 & 0 & 0 & 0 & 0 & 0\end{array}\right)$ & $\left(\begin{array}{l}0 \\
0 \\
0\end{array}\right)$ & $\begin{array}{l}52,5 m, \overline{5} m \\
n 22, n m m,[\bar{n} m 2, \bar{n} 2 m], n / m m m, \\
\text { where } n=8,10 \text { or } 12\end{array}$ \\
\hline $\begin{array}{l}5 m, 5 m^{\prime}, 5 m 1^{\prime} \\
n m m, n m^{\prime} m^{\prime},\left[n^{\prime} m^{\prime} m, n^{\prime} m m^{\prime}\right], n m m 1^{\prime}, \\
\text { where } n=8,10 \text { or } 12\end{array}$ & $\left(\begin{array}{cccccc}0 & 0 & 0 & 0 & d_{15} & 0 \\
0 & 0 & 0 & d_{15} & 0 & 0 \\
d_{31} & d_{31} & d_{33} & 0 & 0 & 0\end{array}\right)$ & $\left(\begin{array}{l}0 \\
0 \\
p\end{array}\right)$ & $\begin{array}{l}52^{\prime}, 5 m^{\prime}, \overline{5} m^{\prime} \\
n 2^{\prime} 2^{\prime}, n m^{\prime} m^{\prime},\left[\bar{n} m^{\prime} 2^{\prime}, \bar{n} 2^{\prime} m^{\prime}\right], n / m m^{\prime} m^{\prime} \\
\text { where } n=8,10 \text { or } 12\end{array}$ \\
\hline
\end{tabular}

Table 3

The point groups of quasicrystals with non-vanishing magnetoelectric effect.

The groups within brackets differ only by their orientation. The effect vanishes for the pentagonal, octagonal, decagonal, dodecagonal and icosahedral groups that do not appear in the table.

\begin{tabular}{|c|c|}
\hline Point group & $\begin{array}{l}\text { Form of the matrix describin } \\
\text { the magnetoelectric effect }\end{array}$ \\
\hline $\begin{array}{l}5, \overline{5}^{\prime} \\
n, \bar{n}^{\prime}, n / m^{\prime} \\
\text { where } n=8,10 \text { or } 12\end{array}$ & $\left(\begin{array}{ccc}\alpha_{11} & \alpha_{12} & 0 \\
-\alpha_{12} & \alpha_{11} & 0 \\
0 & 0 & \alpha_{33}\end{array}\right)$ \\
\hline $\begin{array}{l}52,5 m^{\prime}, \overline{5}^{\prime} m^{\prime} \\
n 22, n m^{\prime} m^{\prime},\left[\bar{n}^{\prime} m^{\prime} 2, \bar{n}^{\prime} 2 m^{\prime}\right], n / m^{\prime} m^{\prime} m^{\prime}, \\
\text { where } n=8,10 \text { or } 12\end{array}$ & $\left(\begin{array}{ccc}\alpha_{11} & 0 & 0 \\
0 & \alpha_{11} & 0 \\
0 & 0 & \alpha_{33}\end{array}\right)$ \\
\hline $\begin{array}{l}5 m, 52^{\prime}, \overline{5}^{\prime} m \\
n m m, n 2^{\prime} 2^{\prime},\left[\bar{n}^{\prime} m 2^{\prime}, \bar{n}^{\prime} 2^{\prime} m\right], n / m^{\prime} m m \\
\text { where } n=8,10 \text { or } 12\end{array}$ & $\left(\begin{array}{ccc}0 & \alpha_{12} & 0 \\
-\alpha_{12} & 0 & 0 \\
0 & 0 & 0\end{array}\right)$ \\
\hline $235, m^{\prime} \overline{3}^{\prime} \overline{5}^{\prime}$ & $\left(\begin{array}{ccc}\alpha_{11} & 0 & 0 \\
0 & \alpha_{11} & 0 \\
0 & 0 & \alpha_{11}\end{array}\right)$ \\
\hline
\end{tabular}

for the 11 crystallographic Laue classes were first determined by Fumi (1951, 1952) (see also Fumi, 1987). The result is reproduced in chapter 2.1.4 of Landolt-Börnstein (1979) as Table 6 and 'Definitions according to Brugger' of Table 7. Because a fourfold and a sixfold rotation along the same axis generates a twelvefold rotation along that axis, the form of $c_{\lambda \mu \nu}$ for $\mathrm{L} 12_{1}$ is found by determining $c_{\lambda \mu \nu}$ such that it satisfies the restrictions for 4 and for 6 . The result is that $c_{\lambda \mu \nu}$ satisfying the restrictions for 6 satisfies those for 12 if $c_{222}=c_{111}$ and $c_{116}=$ 0 , which reduces the number of independent constants from 12 to 10 . Similarly, the form for $\mathrm{L} 12_{2}$ is found by determining $c_{\lambda \mu \nu}$ such that it satisfies the restrictions for 422 and 622 . The result is that $c_{\lambda \mu \nu}$ satisfying the restrictions for 622 satisfies those for
1222 if $c_{222}=c_{111}$, which reduces the number of independent constants from 10 to 9 . This form with nine independent constants is mentioned in chapter 2.1.4 of Landolt-Börnstein (1979) as the one valid for transversely isotropic materials, which misses the point that the forms for $\mathrm{L} 12_{1}$ and $\mathrm{L} 12_{2}$ are not the same for the third-order elastic coefficients. Notice that the form for $\mathrm{L}_{1} 2_{i}$ is valid for all $\mathrm{L} n_{i}$ with $n \geq 7(i=1,2)$ because we deal with a tensor of rank 6 with positive parity. Using different methods, Rama Mohana Rao \& Hemagiri Rao (1993) considered the third-order elastic stiffnesses for the two icosahedral, five pentagonal and two ( $\overline{10}$ and $\overline{10} \mathrm{~m} 2)$ of the seven decagonal point groups. As expected, their result for $\overline{10}$ agrees with ours for $\mathrm{L}_{1}$ and their result for $\overline{10} \mathrm{~m} 2$ with ours for $\mathrm{L} n_{2}, n \geq 7$. Notice that their results for the pentagonal point groups differ from transverse isotropy and their result for the icosahedral point groups differs from isotropy, and that they cannot be obtained simply by applying our general results to the forms given by Fumi (1951, 1952).

Rama Mohana Rao \& Hemagiri Rao (1993) considered also the second-order piezomagnetic effect, i.e. magnetization as a quadratic function of the stresses. Magnetization being described by an axial vector, the effect is described by an axial tensor of rank 5. The authors consider the form of this tensor only for the point groups mentioned above, which makes little sense because the effect depends on magnetic order, i.e. on the Heesch-Shubnikov point group of the crystal. It would make more sense to consider the second-order piezoelectric effect, which is more easily measured experimentally. Its form does not depend on magnetic order; it is described by a polar tensor of rank 5 , i.e. a tensor of negative parity.

\section{The form of tensors describing magnetic properties of quasicrystals; comparison with literature results}

We showed that for quasicrystals there are three nonvanishing forms of the piezoelectric tensor. This tensor is invariant under time reversal $1^{\prime}$, figuratively under the 
Table 4

Heesch-Shubnikov point group symmetries and the corresponding forms of property tensors behaving under inversions in the various possible ways.

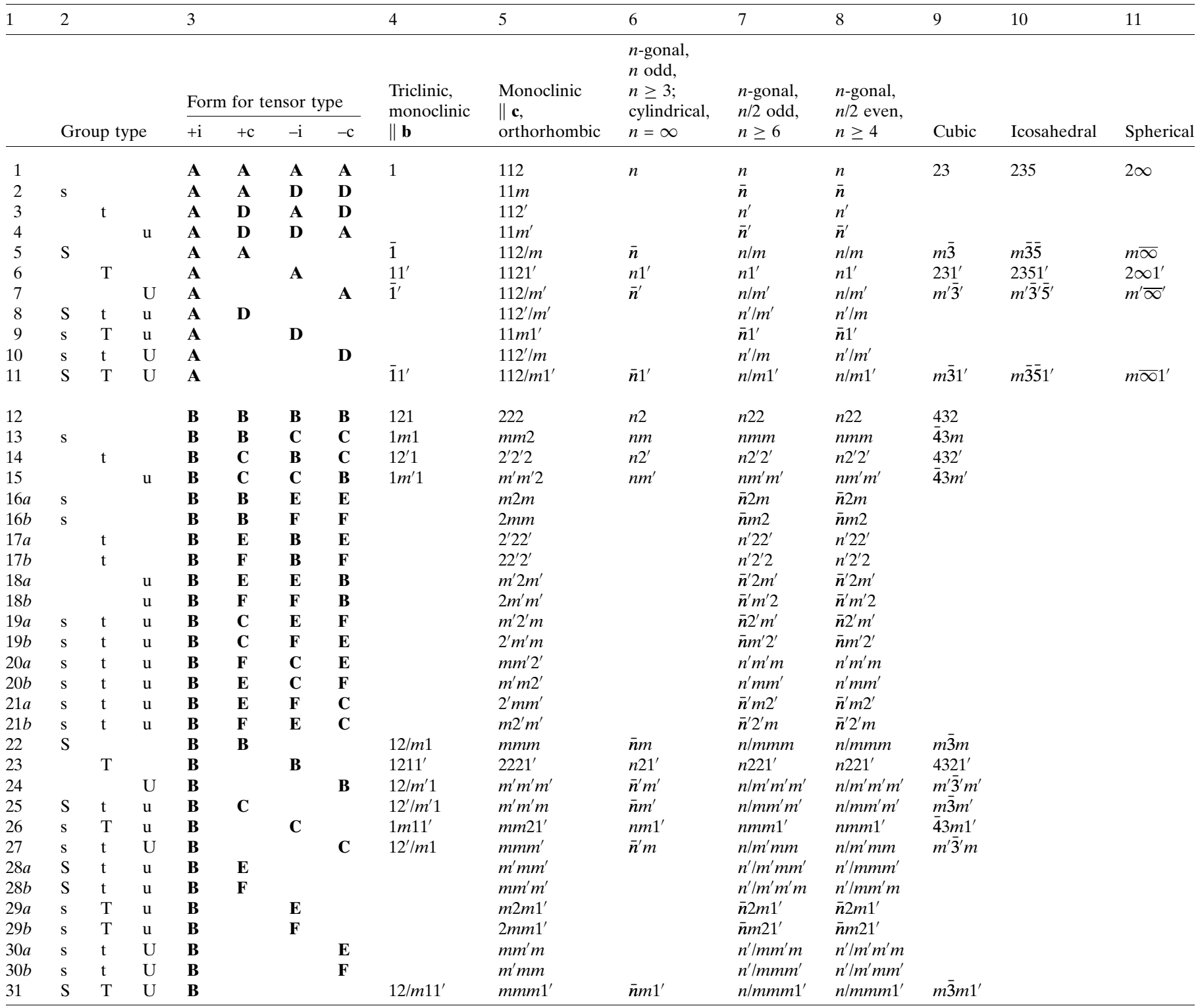

exchange of black and white. The first group in each row of column 1 in Table 2 is monochrome, the last is the corresponding grey group, i.e. the direct product of the monochrome group and the group $1^{\prime}=\left\{1,1^{\prime}\right\}$. The remaining groups of each row are the black-white subgroups of index 2 of the grey group. Whereas the piezoelectric effect is invariant under time reversal $1^{\prime}$ and changes sign under space inversion $\overline{1}$, the piezomagnetic effect is invariant under $\overline{1}$ and changes sign under $1^{\prime}$. It then follows from Neumann's principle that a symmetry operation $\bar{n}$ imposes the same restrictions on the piezoelectric tensor as a symmetry operation $n^{\prime}$ imposes on the piezomagnetic tensor. Taking into account that the mirror reflection $m$ is the same as $\overline{2}$, we can write down also for piezomagnetism the quasicrystal symmetries corresponding to the various forms of the $3 \times 6$ matrix, as shown in column 4 of
Table 2. The last group in each row of column 4 is centrosymmetric; the others are its non-centrosymmetric subgroups of index 2 .

The vector describing pyroelectricity shows the same behaviour under $\overline{1}$ and $1^{\prime}$ as the tensor describing piezoelectricity; also pyromagnetism and piezomagnetism show the same behaviour under $\overline{1}$ and $1^{\prime}$. Nye (1985) showed that the vector describing pyroelectricity has the form

$T[3]=\left(\begin{array}{l}0 \\ 0 \\ p\end{array}\right), \quad T[32]=\left(\begin{array}{l}0 \\ 0 \\ 0\end{array}\right), \quad$ whence $T[3 m]=\left(\begin{array}{l}0 \\ 0 \\ p\end{array}\right)$.

Because of Hermann's theorem, similar results are obtained if the threefold rotation is replaced by an $n$-fold rotation $\| z$ with $n \geq 2$. The result is given in Table 2 . 
Table 5

Monochrome point groups and the corresponding forms of non-magnetic property tensors with parity + or - .

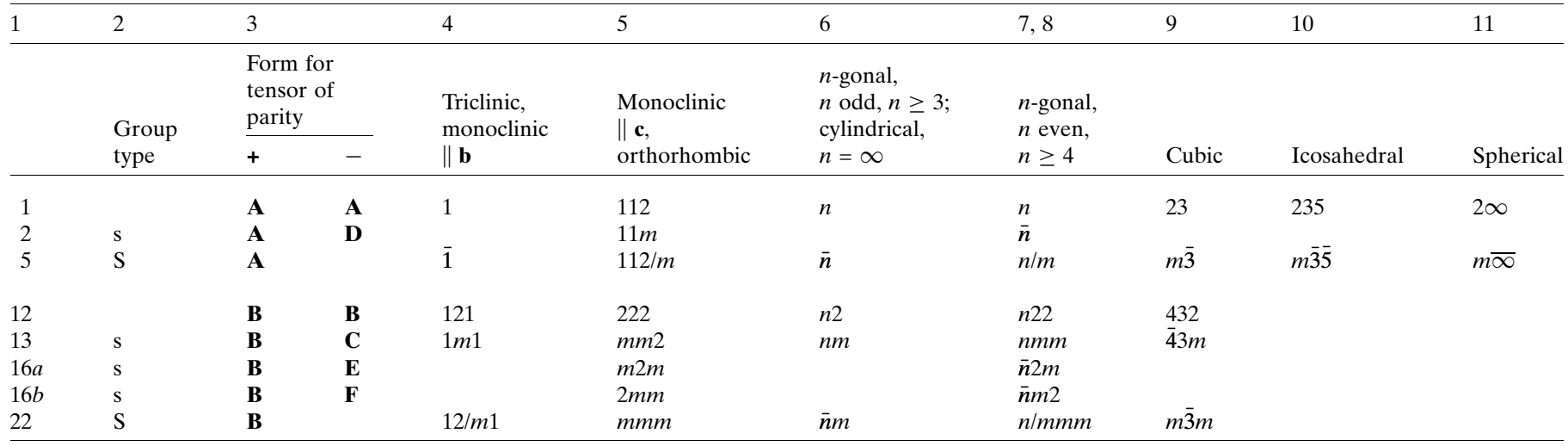

Rama Mohana Rao \& Hemagiri Rao (1992) determined the possible forms of the matrix describing piezomagnetism and of the vector describing pyromagnetism in a different way for the icosahedral, pentagonal and some decagonal point groups. Notice that in their Tables 4-6 the Hermann-Mauguin symbol should be $\overline{5} m$ or $\overline{5} 2 / m$ instead of $\overline{5} 2 m ; \overline{5} m^{\prime}$ or $\overline{5} 2^{\prime} / m^{\prime}$ instead of $\overline{5} 2^{\prime} m^{\prime} ; \overline{5}^{\prime} m^{\prime}$ or $\overline{5}^{\prime} 2 / m^{\prime}$ instead of $\overline{5}^{\prime} 2 m^{\prime} ; \overline{5}^{\prime} m$ or $\overline{5}^{\prime} 2^{\prime} / m$ instead of $\overline{5}^{\prime} 2^{\prime} m$.

The magnetoelectric effect describes the electric polarization produced in matter by a magnetic field applied to it, and the magnetization produced by an electric field: $P_{i}=\alpha_{i j} H_{j}, M_{i}=$ $\alpha_{j i} E_{j}$. Thermodynamics tells us that the two effects are described by transposed matrices. $\mathbf{P}$ and $\mathbf{E}$ change sign under $\overline{1}$ and are invariant under $1^{\prime} ; \mathbf{M}$ and $\mathbf{H}$ are invariant under $\overline{1}$ and change sign under $1^{\prime}$. It follows that $\alpha$ changes sign under $\overline{1}$ and $1^{\prime}$ but is invariant under $\overline{1}^{\prime}$. The rank of the tensor $\alpha$ is 2 , i.e. symmetry rotations of any order $n>2$ have the same effect. It follows that the forms of the tensor for the trigonal groups are valid also for the corresponding pentagonal groups, the forms for the hexagonal groups also for the corresponding octagonal, decagonal and dodecagonal groups, the forms for the groups in the Laue class containing 23 also for the corresponding icosahedral groups. The forms for the trigonal, hexagonal and cubic groups can be found e.g. in BorovikRomanov \& Grimmer (2003); the corresponding results for the point groups of quasicrystals are given in Table 3 .

Rama Mohana Rao \& Hemagiri Rao (1992) determined the possible forms of the magnetoelectric tensor for the icosahedral, pentagonal and some decagonal point groups in a different way. Notice that a minus sign is missing in their result for $5 m$.

\section{Heesch-Shubnikov point-group symmetries; general results on the form of property tensors}

A property tensor may have parity + or - and be invariant (i) or change sign (c) under time reversal. In the previous sections, examples have been given for all four possible combinations: Hall effect $+\mathrm{i}$, pyro- and piezomagnetism $+\mathrm{c}$, pyro- and piezoelectricity $-\mathrm{i}$, magnetoelectric effect $-\mathrm{c}$.
For icosahedral symmetries, a property tensor of given rank and internal symmetry has either the same form as for 235 or it vanishes. We refer to this standard form $T[235]$ as A. A property tensor of type $+\mathrm{i},+\mathrm{c},-\mathrm{i}$ or $-\mathrm{c}$ has the form $\mathbf{A}$ if column 10 of Table 4 contains an $\mathbf{A}$ in the row containing the icosahedral group under consideration, otherwise the tensor will vanish.

For spherical symmetries, a property tensor of given rank and internal symmetry has either the same form as for $2 \infty$ or it vanishes. We refer to this standard form $T[2 \infty]$ as A. A property tensor of type $+\mathrm{i},+\mathrm{c},-\mathrm{i}$ or $-\mathrm{c}$ has the form $\mathbf{A}$ if column 11 of Table 4 contains an $\mathbf{A}$ in the row containing the icosahedral group under consideration, otherwise the tensor will vanish.

For the $n$-gonal groups with $n \geq 3$, we choose the axis c along the principal symmetry direction, $\mathbf{a}$ and $\mathbf{b}$ in the plane normal to $\mathbf{c}$ at an angle of $[(n-1) / n] \times 180^{\circ}$ if $n$ is odd, $[(n-2) / n] \times 180^{\circ}$ if $n$ is even. Adopting the usual conventions on the symmetry directions corresponding to the entries in the Hermann-Mauguin symbol: first entry along $\mathbf{c}$, second along a, third along $\mathbf{a}-\mathbf{b}$, it follows that $\mathbf{a}-\mathbf{b}$ makes an angle of $180^{\circ} / n$ with a if $n$ is even, $90^{\circ} / n$ if $n$ is odd. (Notice that only the first two symmetry directions appear in the HermannMauguin symbol if $n$ is odd.)

For $n$-gonal symmetry with $n$ odd, a property tensor of given rank and internal symmetry has either the form $\mathbf{A}=T[n], \mathbf{B}=$ $T[n 2], \mathbf{C}=\mathbf{A} \ominus \mathbf{B}$ (which means $\mathbf{A}=\mathbf{B} \oplus \mathbf{C}$ ), or it vanishes. A property tensor of type $+\mathrm{i},+\mathrm{c},-\mathrm{i}$ or $-\mathrm{c}$ has form $\mathbf{A}, \mathbf{B}$ or $\mathbf{C}$, respectively, if column 6 of Table 4 contains that letter in the row containing the group under consideration, otherwise the tensor will vanish. The same is true for cylindrical symmetry, in which case $n=\infty$.

For $n$-gonal symmetry with $n=2 k$ even, a property tensor of given rank and internal symmetry has either the form $\mathbf{A}=$ $T[n], \mathbf{B}=T[n 22], \mathbf{C}=\mathbf{A} \ominus \mathbf{B}, \mathbf{D}=T[k] \ominus \mathbf{A}, \mathbf{E}=T[k 22] \ominus \mathbf{B}$, $\mathbf{F}=\mathbf{D} \ominus \mathbf{E}=\{T[k] \ominus T[k 22]\} \ominus \mathbf{C}$ or it vanishes. A property tensor of type $+\mathrm{i},+\mathrm{c},-\mathrm{i}$ or $-\mathrm{c}$ has one of the forms $\mathbf{A}$ to $\mathbf{F}$ if column 7 (for $k$ odd) or column 8 (for $k$ even) of Table 4 contains that letter in the row containing the group under consideration, otherwise the tensor will vanish. 
A letter $\mathrm{S}$ in the column 'group type' of Table 4 states that the groups in the corresponding row contain space inversion $\overline{1}$, a $T$ that they contain time reversal $1^{\prime}$, a $U$ that they contain space-time inversion $\overline{1}^{\prime}$. The lower case letters $s, t$ and $u$ state that the groups contain the corresponding inversions only combined with non-trivial rotations.

Table 4 extends Table 2 of Grimmer (1991) (see also Grimmer, 2006) not only to quasicrystalline point groups but also to all groups considered in Table 10.1.4.2 of Hahn \& Klapper (2002).

\section{Discussion and conclusions}

Using a theorem due to Carl Hermann, we have shown how the restrictions on the form of property tensors of rank $<5$ that follow from the Neumann principle for the point groups describing quasicrystals can be deduced from the restrictions for the point groups describing crystals. We showed this explicitly for the icosahedral and $n$-gonal point groups, where $n=5,8,10$ or 12 ; obviously the arguments given apply also for any $n>6$. It follows that in Tables 2 and 3 one may replace in the point group symbols 5 by $n$ and add ' $n$ odd, $n \geq 5$ ', and one may replace ' $n=8,10$ or 12 ' by ' $n$ even, $n \geq 8$ '. For octagonal and dodecagonal point groups, the restrictions on the form of property tensors can be deduced from those for the point groups describing crystals even for tensors of rank $<8$ and $<12$, respectively, as exemplified by our discussion of the thirdorder elastic stiffnesses.

The results derived in a number of papers for various physical properties of quasicrystals with certain point-group symmetries have been generalized to all quasicrystal point groups. It has been shown that the non-standard classification of quasicrystal point groups with a principal axis into pentagonal, decagonal, octagonal and dodecagonal ones used in those papers obscures general features of their results.

Table 4, which is valid for property tensors of arbitrary rank, simplifies considerably for 'non-magnetic' properties, i.e. properties that are invariant under time reversal. In this case, possible magnetic order can be neglected in determining the point group, which, for simplicity, is taken as monochrome, not grey. The result is given as Table 5 .

We showed that it follows from the theorem of Hermann (1934) that $\mathbf{D}, \mathbf{E}$ and $\mathbf{F}$ vanish for $n$-gonal point groups if the rank of the property tensor is less than $n / 2$. For cylindrical point groups, we conclude that this is true for property tensors of arbitrary rank. Notice that this is automatically taken into account by treating $\infty$ as an odd number.
It is interesting to compare our results with those of Janssen (2003). He considers the piezoelectric and elastic properties of quasicrystals making use of tensors in superspace. As examples, he determines the piezoelectric tensor for an octagonal quasicrystal with point group $8 \mathrm{~mm}$ and the elastic tensor for an icosahedral quasicrystal with point group 235. Comparing his results with ours suggests that applying Neumann's principle in our physical subspace of his superspace yields what Janssen calls the phonon part of the tensor. This supposition is fully confirmed by the results of $\mathrm{Hu}$ et al. (2000).

\section{References}

Borovik-Romanov, A. S. \& Grimmer, H. (2003). International Tables for Crystallography, Vol. D, edited by A. Authier, pp. 105-149. Dordrecht: Kluwer Academic Publishers

Fumi, F. G. (1951). Phys. Rev. 83, 1274-1275.

Fumi, F. G. (1952). Phys. Rev. 86, 561.

Fumi, F. G. (1987). Acta Cryst. A43, 587-588.

Grimmer, H. (1991). Acta Cryst. A47, 226-232.

Grimmer, H. (1993). Acta Cryst. A49, 763-771.

Grimmer, H. (2006). http://www.lcm3b.uhp-nancy.fr/mathcryst/pdf/ Leuven_Grimmer.pdf

Hahn, Th. \& Klapper, H. (2002). International Tables for Crystallography, Vol. A, 5th ed., edited by Th. Hahn, pp. 761-808. Dordrecht: Kluwer Academic Publishers.

Hermann, C. (1934). Z. Kristallogr. 89, 32-48.

Hu, C. Z., Wang, R. H. \& Ding, D. H. (2000). Rep. Prog. Phys. 63, 1-39.

Janssen, T. (2003). International Tables for Crystallography, Vol. D, edited by A. Authier, pp. 243-264. Dordrecht: Kluwer Academic Publishers.

Jiang, Y. J., Liao, L. J. \& Chen, G. (1995). Acta Cryst. A51, 159-163.

Jiang, Y. J., Liao, L. J., Chen, G. \& Shen, J. J. (1992). Acta Cryst. A48, 350-352.

Jiang, Y. J., Liao, L. J., Chen, G. \& Zhang, P. X. (1990). Acta Cryst. A46, 772-776.

Landolt-Börnstein (1979). Numerical Data and Functional Relationships in Science and Technology. New Series, Group III: Crystal and Solid State Physics, Vol. 11, Elastic, Piezoelectric, Pyroelectric, Piezooptic, Electrooptic Constants, and Nonlinear Dielectric Susceptibilities of Crystals. Berlin: Springer.

Nye, J. F. (1985). Physical Properties of Crystals: their Representation by Tensors and Matrices. Oxford: Clarendon Press.

Rama Mohana Rao, K. \& Hemagiri Rao, P. (1992). J. Phys. Condens. Matter, 4, 5997-6008.

Rama Mohana Rao, K. \& Hemagiri Rao, P. (1993). J. Phys. Condens. Matter, 5, 5513-5524.

Steurer, W. (2004). Z. Kristallogr. 219, 391-446.

Walpole, L. J. (1984). Proc. R. Soc. London Ser. A, 391, 149-179. 